\title{
Lithium Carbonate
}

National Cancer Institute

\section{Source}

National Cancer Institute. Lithium Carbonate. NCI Thesaurus. Code C1318.

The carbonate salt of lithium, a soft alkali metal, with antimanic and hematopoietic activities. Lithium interferes with transmembrane sodium exchange in nerve cells by affecting sodium, potassium-stimulated adenosine triphosphatase ( $\mathrm{Na+}, \mathrm{K}+-$ AT Pase); alters the release of neurotransmitters; affects cyclic adenosine monophosphate (cAMP) concentrations; and blocks inositol metabolism resulting in depletion of cellular inositol and inhibition of phospholipase C-mediated signal transduction. The exact mechanism through which lithium exerts its mood-stabilizing effect has not been established. In addition, lithium stimulates granulocytopoiesis and appears to increase the level of pluripotent hematopoietic stem cells by stimulating the release of hematopoietic cytokines and/or directly acting on hematopoietic stem cells. 